\title{
Thermal Response of helix ground heat exchangers
}

\author{
Marco Fossa
}

\author{
Benoit Stutz
}

\author{
Antonella Priarone
}

Antoine Coperey

\begin{abstract}
This paper is devoted to the thermal analysis of shallow ground heat exchangers with pipes arranged in a helix configuration. The pipes where the carrier fluid is circulated typically embrace a cylindrical volume that is filled by ground or concrete, the latter being the case of the so called geopiles. Other pipes dispositions include conic belices that can be easily inserted in proper excavations. The analysis of the transient thermal behavior of a belix/ground assembly is here carried out according to different approaches, including the exploitation of superposition techniques, the finite element modelling and experiments in a reduced scale mock up. Different geometrical configurations have been taken into account and also the variability of ground and concrete thermal properties have been considered. A detailed description of the experimental set up is provided and the model results have been processed in order to develop suitable temperature response factors (or g-functions) to be employed for predicting the ground heat exchanger behavior in different operating conditions.
\end{abstract}

\section{INTRODUCTION}

Short vertical heat exchangers are often employed in Ground Coupled Heat Pump (GCHPs) applications since they usually do not need specific deep drilling equipment and can be buried on place during the operations of excavation in the building site. Such shallow heat exchangers (compared to deep borehole heat exchangers, BHE) can be either placed horizontally typically within 1 meter of soil or in trenches of various shapes down to some dozens of meters. Trench heat exchangers are often arranged as helix pipes (Helix Heat Exchanger, HHE) occupying a cylindrical volume in the ground that can be either filled with soil or concrete, hence realizing in the latter configuration a cylindrical geopile. When employing shallow heat exchangers, the external diameter of the whole assembly is comparable to the depth of the heat transfer surface. Simple models that describe linear (infinite line source ILS or finite line source FLS, Ingersoll et al., 1954, Eskilson 1987, Lamarche and Beauchamp 2007) and cylindrical infinite heat sources (ICS, Ingersoll et al., 1954) are not suitable for either describing the performance of the heat exchanger to constant and variable heat loads or to infer the ground properties in preliminary in situ tests devoted to soil conductivity evaluation.

In fact, the axial heat conduction contribution in the filled inner part of HHEs becomes not negligible, especially in the long term operations, and significantly affects the thermal response of the system. Moreover, the actual geometry of the HHE, i.e. the helix pitch, influences the temperature field especially close to the carrier fluid pipes. On this subject different studies have been developed during the last decades, either from the analytical or numerical point of view. One of the first pioneering studies is the numerical simplified model of the spiral heat exchanger by Rabin and Korin (1996) in which the Authors represented the behaviour of helix by means of a series of

Marco Fossa (marco.fossa@unige.it) is professor of Solar and Geothermal Energy and Antonella Priarone is research professor, both at DIME, University of Genova, Italy. Benoit Stutz is professor at Université Savoie Mont Blanc, LOCIE - Polytech Annecy-Chambéry, France and Antoine Coperey is PhD student at Locie. 
rings, with the same pitch distance. The effects of the thermal properties of the soil, the aspect ratio of the heat exchanger and its pitch distance have been analysed and compared with experimental data from field experiments.

A group of researchers has more recently developed several analytical solutions based on the Green's function method, with increasing accuracy to represent the thermal response of HHEs into the ground (Man et al. 2010, Cui et al. 2011, Man et al. 2011). Exploiting the possibility of the superposition of basic solutions, they analysed either infinite or finite geometries, the seconds represented by the use of the virtual heat source theory. The starting point was the solution of the infinite and finite "solid" cylindrical model, where the inner space of the helix heat source was filled by ground. In these studies analytical solutions in terms of integrals to be solved have been presented for either ring or helix heat sources.

A few of recent studies combine the pure conduction into the soil surrounding a spiral coil with other mechanisms. Moch et al. (2014) analytically modelled and numerically solved the soil freezing problem around a helix coil, represented as an annular cylinder or a series of rings; the theoretical results have also been compared with experimental data with a satisfactory agreement. Go et al. (2015) analysed the effects of groundwater advection into the ground on the performance of a spiral coil field. Finally, Dehghan et al. (2016) developed fully 3D numerical simulations of spiral coils fields to analyse the effects of the different geometrical parameters including the distance between the heat exchangers, their aspect ratio, and the pitch distance between spires.

Based on the present literature survey it is possible to conclude that additional aspects of the problem deserve further investigation. These aspects include the development of laboratory scale facilities where to infer experimental data on heat exchanger behaviour in controlled conditions. Another issue is related to the development of proper temperature response factors (TRF or $\mathrm{g}$-functions) for the helix source by applying the concepts first developed by Eskilson (1987). Finally the different thermal properties of ground and concrete media (in geopile configurations) have not been taken into consideration and dedicated models need to be developed to this special but not uncommon case.

In this paper the problem of developing temperature response factors for helix shaped ground heat exchangers is tackled according different strategies that include a semi analytical approach based on basic solution spatial superposition and the numerical solution of the 3D conduction equation with FEM codes.

Furthermore an experimental set up for assessing the heat exchanger performance is proposed and a detailed description of the laboratory scale set up for helix heat exchangers is provided.

\section{TEMPERATURE RESPONSE FACTORS WITH SPATIAL SUPERPOSITION}

The solution of the transient conduction equation is often the typical approach to describe the ground thermal response to a system of (or to a single) ground heat exchanger. One-dimensional (in the radial direction) and twodimensional (radial and axial) analytical solutions have been proposed for predicting the ground response to a single constant heat pulse. These base solutions (temperature response factors) can be superposed in time and space (Ingersoll et al. 1954, Eskilson 1987), for describing the transient response of a BHE borefield/ground assembly to any stepwise function describing the variable thermal load to the ground during the seasons (Yavuzturk and Spitler, 1999). Linear heat source solutions (ILS and FLS models) are based on the integration over a line of the Single Point Source solution (SPS, Ingersoll et al., Eq. 1):

$$
T(r, \tau)-T_{g r, \infty}=\frac{\dot{Q}}{4 \pi k_{g r} r} \operatorname{erfc}\left(\frac{1}{2 \sqrt{F o_{r}}}\right)
$$

In the above expression, $\mathrm{T}$ is the ground temperature, $\mathrm{r}$ is the radial distance from the point source, $\mathrm{k}_{\mathrm{gr}}$ is the ground thermal conductivity, $\mathrm{T}_{\mathrm{gr}, \infty}$ is the undisturbed (initial) ground temperature, $\mathrm{Fo}_{\mathrm{r}}$ is the radius based Fourier number and finally $\dot{Q}$ is the heat transfer rate. By performing an integration along a line of length $\mathrm{H}$, the temperature 
field at any radial and axial position around the line source (in infinite medium) can be evaluated as superposed contributions by the multiple point sources.

$$
T(r, z, \tau)-T_{g r, \infty}=\frac{\dot{Q}^{\prime}}{4 \pi k_{g r}} \int_{0}^{H} \frac{\operatorname{erfc}\left(\frac{\sqrt{(z-h)^{2}+r^{2}}}{2 \sqrt{\alpha \tau}}\right)}{\sqrt{(z-h)^{2}+r^{2}}} d h
$$

Once the temperature field in time is known, a dimensionless temperature difference ( $\mathrm{g}$-function) can be obtained based on the concept of temperature response factor and with reference to a given boundary condition (heat rate BC or temperature BC, Priarone and Fossa 2016) and a reference radial distance. Being $\bar{T}$ the average ground temperature at reference distance from (single or multiple) heat source, the temperature response factor can be evaluated as:

$$
g(F o)=2 \pi k_{g} \frac{\bar{T}(\tau)-T_{g r, \infty}}{\dot{Q}_{H}^{\prime}}
$$

When referring to BHEs, the above reference distance is typically the borehole radius. In the present investigation the location at which the average temperature is evaluated is at pipe to solid interface, irrespective of the pipe arrangement in the solid medium.

The approach for calculating the TRF of HHEs is here based on a numerical calculation of the temperature field at radial distance $\mathrm{r}_{\mathrm{p}}$ (Figure 1) from a helix curved line describing the heat exchanger coil. The line of individual sources has been discretized in terms of single SPSs and includes mirror sources for realizing the interface condition of constant temperature at ground top interface. A mesh sensitivity to $g$-function results have been performed with reference to the ILS and FLS reference solutions, as calculated for example in Fossa (2016).

\section{FINITE ELEMENT ANALYSIS OF HELIX TYPE HEAT EXCHANGERS}

A FEM model has been built to further develop the TRF concepts applied to conditions where the HHE inner volume has different thermal properties from the external surrounding soil. The model has been developed within Comsol Multyphysics environment. The model geometry is described in Figure 1. The helix coil is simplified by considering $N$ circular rings with the same pitch distance $p$, with imposed constant heat flux on their surface. Two domains are considered, both represented in axial symmetry coordinates: the inner (made by concrete in case of geopiles) with radius $r_{c}$ and depth $H$ and the external ground volume, with radius $r_{g}$ and depth $H_{g}$. The rings are tubular surfaces of $r_{p}$ radius (pipe radius) and distance from the axis equal to $r_{b}$. An additional dimension has been introduced for accounting for geopile covering thickness at pipe location. This extra thickness of concrete has been set equal to $5 r_{p}$.

Assuming pure conduction in both the concrete and the ground domains, the transient equations to be solved are the Fourier ones, either in terms of ground (g) or concrete (c) properties.

$$
\frac{\partial T}{\partial \tau}=\frac{k_{c}}{\rho c} \nabla^{2}(T)
$$

$\mathrm{T}$ is here the temperature, $\mathrm{t}$ is time, $\mathrm{k}$ is thermal conductivity, $\rho$ is density and $\mathrm{c}$ the specific heat.

The boundary conditions are imposed heat flux on the ring surface (Eq. 5) and imposed temperature $\left(\mathrm{T}=\mathrm{T}_{\mathrm{gr}, \infty}\right)$ on the ground/concrete upper surface and on the external surfaces of the ground domain. 


$$
-k_{c}(\nabla T \cdot \mathbf{n})=\frac{\dot{Q}_{L}^{\prime}}{2 \pi \cdot r_{p}}=\frac{\dot{Q}_{H}^{\prime}}{4 \pi^{2} \cdot r_{p} \cdot \frac{r_{b}}{p}}
$$

$\dot{Q}_{L}^{\prime}$ represents the heat transfer rate for unit length of the spiral pipe, whereas $\dot{Q}_{H}{ }_{H}$ the heat transfer rate for unit depth of the pile. By assuming that the distance of the first and the bottom rings from the end of the pile is $p / 2$, the relationship between the two values is obtained by considering that

$$
\dot{Q}_{L}^{\prime}=\frac{\dot{Q}}{2 \pi \cdot r_{b} \cdot N}=\frac{\dot{Q}}{2 \pi \cdot r_{b} \cdot \frac{H}{p}}=\frac{\dot{Q}_{H}^{\prime}}{2 \pi \cdot \frac{r_{b}}{p}}
$$

Continuity conditions hold at the interface concreate to ground, and the initial condition is $T=T_{g r, \infty}$ on the whole computational domain. $T_{g r, \infty}$ is the undisturbed ground temperature.

The selected dimensions of the ground volume are $r_{g}=200 \mathrm{~m}$ and $H_{g}=200 \mathrm{~m}$. These values assure an approximation of the ideal case of semi-infinite ground with an error of less than the $1 \%$ for the external boundary temperature with respect to the undisturbed ground temperature.

Different meshes have been tested, with increasing number of elements on the pipes surface and on the interface between the concrete and the ground, up to values that ensure the results in term of average temperature on pipe to not change more, with an accuracy of $0.2 \%$. Finally, an unstructured triangular mesh is chosen, with 12 uniformly spaced elements on each pipe boundary and 450 elements on the interface boundary, for an overall number of elements equal to about 56800 .

The average temperature on the rings surface has been evaluated by simulations as a function of time to infer the dimensionless thermal response factor:

\section{EXPERIMENTAL SET UP FOR REDUCED SCALE HELIX HEAT EXCHANGERS}

An experimental device has been designed and realised at Locie (Université Savoie Mont Blanc) to study the unsteady coupled heat and mass transfers (due to groundwater circulation and even freezing) occurring around a reduced scale HHE under controlled conditions. The experimental setup will be beneficial for assessing the limits of the modelling approach, which is based on thermal properties constant in time and no moisture or water effects. Temperature measurements using thermocouples and water content sensors are employed together with geophysical methods including Direct Current resistivity, Induced Polarization and Self-Potential measurements (Hermans et al 2014, Ikard and Revil 2014). The setup consists of a cylindrical tank (1.0 m diameter; $1.2 \mathrm{~m}$ height) filled with silica sand (grain size $180 \mu \mathrm{m}$ ) of known thermal properties (Figure 2). The side wall is thermally insulated and the tank bottom part is held at constant temperature thanks to a spiral heat exchanger (\#6, Figure 2). A ventilation system controls air temperature and relative humidity on the upper surface of the sand. The presence of a water table is imposed in the set up by acting on a series of valves. Water percolation related to rainfalls is simulated by injecting water at the top of tank. 40 thermocouples (\#14, Figure 2) are vertically distributed into the sand volume at three radial profiles (at $0,5,10 \mathrm{~cm}$ to tank axis) and three vertical positions. Distribution of water content is record by four sensors. Two X-shape profiles of 17 electrodes are set into sand and under the HHE, and four vertical profiles of 28 electrodes are located on tank walls for geophysical measurements in time domain. Set up dimensions have been chosen based on a dimensional analysis using the Buckingham-Pi theorem which reveals scaling laws and remarkable properties of this problem. When soil freezing is neglected, the dimensional analysis show that thermal perturbation of the soil can be characterized in terms of Biot and Fourier numbers together with proper dimensionless geometrical parameters, as defined in Figure 1 and where $\mathrm{D}=2 \mathrm{r}_{\mathrm{b}}$. 

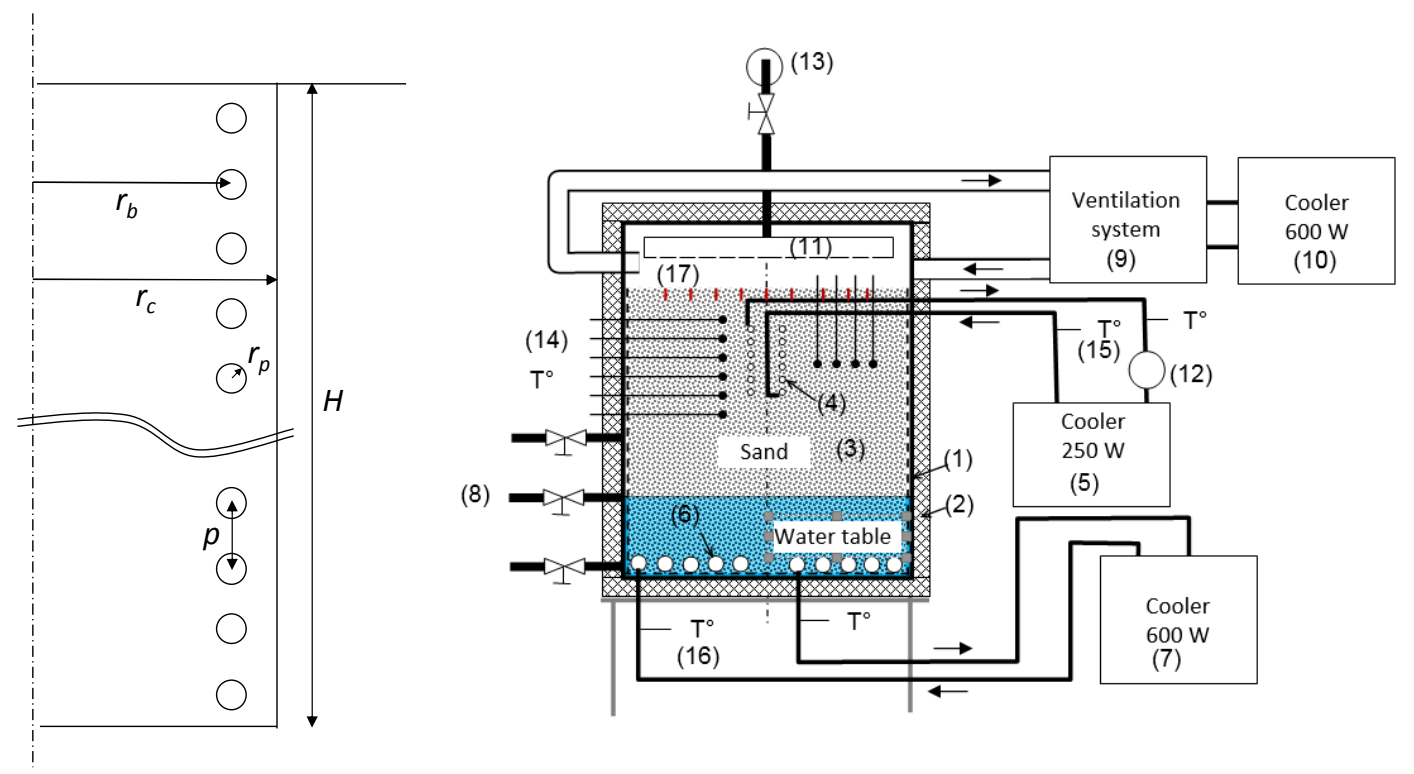

Figure 1. Geometrical parameters in HHE concept
Figure 2. Experimental setup at Locie for HHE characterization. (1) Tank, (2) thermal insulation, (3) Sand volume, (4) HHE, (6) spiral heat exchanger, (11) rain system, (14 to 16) temperature sensors, (17) electrodes

$$
B i=A \cdot\left(F o_{D}\right)^{a} \cdot\left(\frac{H}{D}\right)^{b} \cdot\left(\frac{p}{D}\right)^{c} \cdot\left(\frac{p}{d_{p}}\right)^{d}
$$

Where $\mathrm{Bi}$ is based on thermal power extracted by HHE and the temperature difference between pipe periphery and undisturbed ground temperature.

In this experiment, the reducing scaling factor is 10 for the geometric parameters. The ground properties are conserved as well as the characteristic temperature differences of the problem. The capillary fringe is not scaled (sand grain and pore scales have been not changed). The conservation of the Biot and the Fourier number leads to a reduction by a factor 100 of the time scale (one real year is equal to 3.6 days), and a reduction by a factor 10 of the thermal power extracted by the exchanger. Due to geometrical constraints and based on previous considerations (Moch et al. 2014), it has been decided to scale only the shape parameter H/D.

Carrier fluid temperature is controlled in order to keep constant the pipe outer surface temperature.

\section{RESULTS AND DISCUSSION}

Two different models have been developed as described in previous chapters. Validation of both models has been carried out with reference to available literature solutions, namely the ICS and ILS solutions (Ingersoll et al.) and the FLS solution as proposed by Lamarche and Beauchamp.

The HHE geometry is defined in terms of quantities $\mathrm{H}=15 \mathrm{~m}, \mathrm{r}_{\mathrm{b}}=0.3 \mathrm{~m}, \mathrm{r}_{\mathrm{p}}=0.016 \mathrm{~m}, \mathrm{p}=0.1 \mathrm{~m}$.

Figure 3 shows the HHE/ground response in terms of TRF (Eq. 3) for different values of discretization parameter zstep $/ r_{p}$, where zstep is the distance between single point sources and $r_{p}$ is the position at which the SPS $g$ function is evaluated. Abscissa is the $\mathrm{H}$ based Fourier number $\mathrm{FOH}_{\text {. }}$ As can be observed the SPS solution approaches the reference FLS one when the discretization parameter $z s t e p / r_{p}$ is of the order of the unit and in all calculations has been set equal to 2 , as a compromise choice between accuracy and calculation speed. 


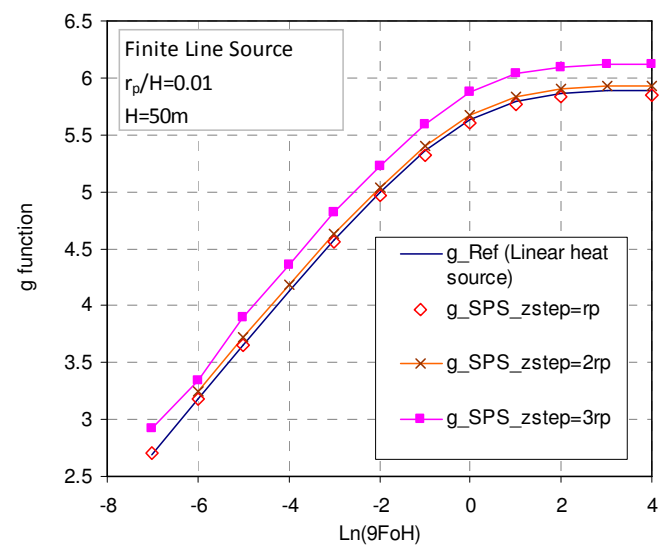

Figure 3. Linear source discretization and sensitivity analysis. Comparison of reference FLS results with SPS superposition solutions.

Parameter: SPS dimensionless distance $z_{\text {step }} / r_{p}$

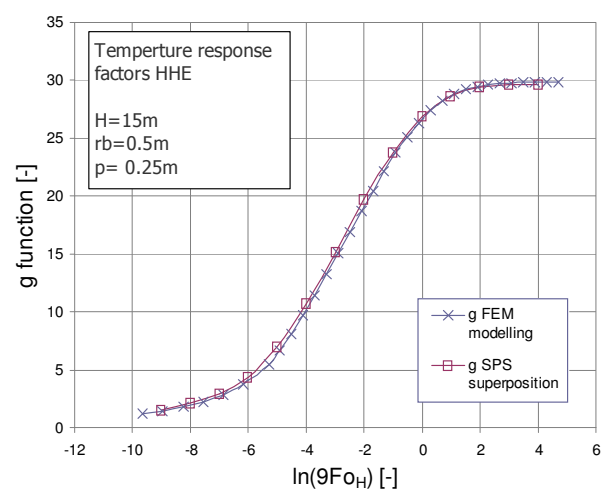

Figure 5. Comparison of SPS superposition and FEM model results in terms of HHE g-function

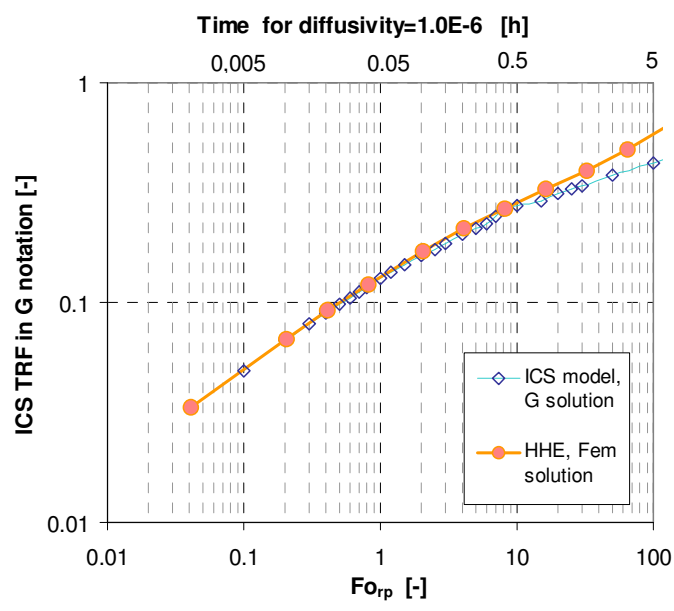

Figure 4. FEM model validation with respect to the reference ICS solution. $\mathrm{H}=15 \mathrm{~m}, \mathrm{r}_{\mathrm{p}}=0.016 \mathrm{~m}$

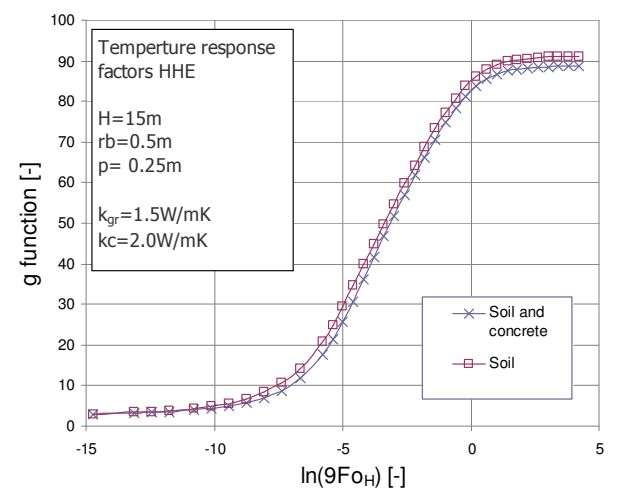

Figure 6. Effects of different ground and concrete thermal properties on HHE g-function (FEM model)

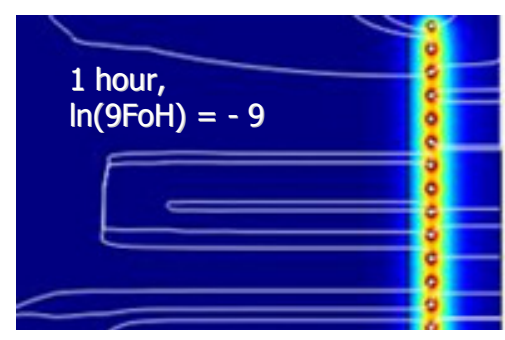

(a)

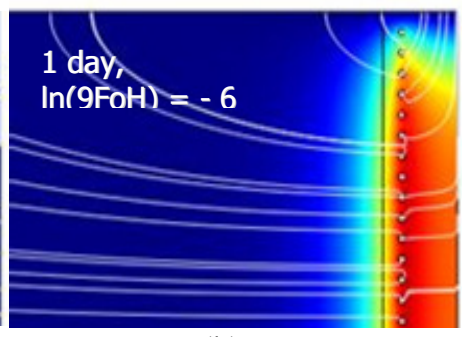

(b)

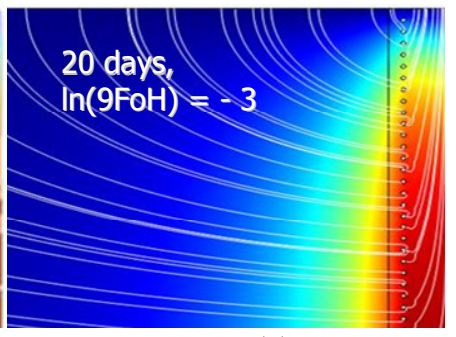

(c)

Figure 7. FEM temperature field around a HHE with different thermal properties in outer (ground) and inner (concrete) volumes

FEM model has been tuned in terms of mesh characteristics either with respect to reference analytical solutions (the ICS model, G solution) or with respect to the SPS superposition model itself. Figure 4 shows the FEM gfunction solution for the HHE source when both the inner and outer parts of the helix surface have the same thermal 
properties (i.e. ground either inside or outside). The average ground temperature $\bar{T}$ is evaluated along the radius $\mathrm{r}_{\mathrm{b}}$. As it could be expected, in the early period the HHE source behaves like the ICS since the mutual influence of neighbour coils and even the ground top interface does not yet play its role.

Figure 5 is the comparison between the SPS and FEM models for the same HHE geometry and medium thermal conductivity $\left(\mathrm{k}=\mathrm{k}_{\mathrm{gr}}=1.5 \mathrm{~W} / \mathrm{mK}\right)$. It can be noticed that the simple 1D solution by SPS superposition is able to describe the HHE response in very good agreement with reference to the FEM solution and hence seems suitable for example for parametric analyses aimed at HHE geometry optimization. Figure 6 refers to FEM different simulations where at given HHE geometry the inner volume of coils has been changed in terms of heat conductivity $\left(\mathrm{k}_{\mathrm{c}}=2.0 \mathrm{~W} / \mathrm{mK}, \mathrm{k}_{\mathrm{gr}}=1.5 \mathrm{~W} / \mathrm{mK}\right)$ while keeping constant both media density and specific heat. The curve named "soil" refers to homogeneous medium $\left(\mathrm{k}=\mathrm{k}_{\mathrm{gr}}\right)$ inside and outside the helix coil while the curve "soil and concrete" is related to a geometry with a concrete inner core. Being the thermal conductivity values quite similar, the two g-function profiles are close each other and almost superpose in the early period. Later in time and toward the asymptotic region the axial conduction along the pile starts to increase the overall heat transfer thanks to a better conductivity of the inner core. To this aim Figures 7 show snapshots of the temperature field at three different instants from heat generation start. In particular image 7 a refers to the early period $\left(\ln \left(9 \mathrm{FOH}_{\mathrm{H}}=-9\right)\right.$ when the heat streamlines are oriented radially and the ground medium offers a convenient heat path. Figures $7 \mathrm{~b}$ and $7 \mathrm{c}$ ( $\ln \left(9 \mathrm{FO}_{\mathrm{H}}\right)$ equal to -6 and -3 respectively) show on the other hand the heat flow through the pipe core, which can take advantage from pile core higher conductivity.

\section{CONCLUSIONS}

In this paper a joint research has been carried out for assessing the performance of trench heat exchangers for geothermal heat pump applications. In particular the helix geometry has been taken into consideration either from an experimental or theoretical point of view. An experimental setup has been conceived and realized at Locie lab in France and data collection is expected to start in 2017, thus allowing a complete comparison between model predictions and real condition HHE thermal response, also in terms of presence of groundwater circulation.

Modeling has been addressed to pure conduction conditions and 1D and 2D models have been proposed. In particular the present 1D model is based on a simple approach of solution superposition starting from the single point source (SPS) while FEM calculations have been performed for taking into account the possibility that the inner volume of helix heat exchanger could be filled by concrete, thus realizing a geopile heat exchanger. Validation of both models with respect to literature solutions showed that the SPS is able to fully describe the geometry effects of HHE parameters and it is hence useful for fast geometry optimization analyses. The FEM model on the other hand is able to cope with different thermal properties inside and outside the helix pipe surface. Cross comparison between models provides first information on the reliability of homogeneous property hypothesis and SPS model application. The temperature response factors (here calculated according to different levels of detail) are considered helpful for applying temporal superposition techniques devoted to variable heat rate simulations of concrete geopiles and helix heat exchangers.

\section{ACKNOWLEDGMENTS}

The Authors acknowledge Dr. Alessandro Cavalletti for his help in FEM calculations he performed during the development of his Thesis at the University of Genova. 


\section{NOMENCLATURE}

$\begin{array}{rll}c & =\text { specific heat } & (\mathrm{J} / \mathrm{kg} \mathrm{K}) \\ F_{o_{r p}}=\text { Fourier number, } \mathrm{Fo}=(\alpha \tau) / r_{p}^{2} & (-) \\ G=\text { ICS temperature response factor } & (-) \\ H=\text { HHE depth } & (\mathrm{m}) \\ k=\text { Thermal conductivity } & (\mathrm{W} / \mathrm{mK}) \\ \dot{Q}=\text { Heat transfer rate } & (\mathrm{W}) \\ \dot{Q}^{\prime} & =\text { Heat transfer rate per unit length } & (\mathrm{W} / \mathrm{m}) \\ p & =\text { coil pich } & (\mathrm{m}) \\ r & =\text { radius } & (\mathrm{m}) \\ T & =\text { Temperature } & \left({ }^{\circ} \mathrm{Cor} \mathrm{K}\right) \\ \alpha & =\text { Thermal diffusivity } & \left(\mathrm{m}^{2} / \mathrm{s}\right) \\ \rho & =\text { density } & \left(\mathrm{kg} / \mathrm{m}^{3}\right) \\ \tau & =\text { Time } & (\mathrm{s})\end{array}$

\section{Subscripts}

$\infty=$ undisturbed conditions

$b \quad=$ HHE radius

$c=$ concrete

gr $\quad=$ ground

$p \quad=$ pipe

\section{REFERENCES}

Carslaw, H.S. and J.C. Jaeger. 1947. Conduction of Heat in Solids, Claremore Press, Oxford, U.K.

Cui, P., X. Li, Y. Man and Z. Fang. 2011. Heat transfer analysis of pile geothermal heat exchangers with spiral coils, Applied Energy 88: 4113-4119.

Dehghan, B., A. Sisman and M. Aydin. 2016. Parametric investigation on helical ground heat exchangers for heat pump applications, Energy and Buildings 127: 999-1007.

Eskilson P., 1987. Thermal Analysis of Heat Extraction Boreholes. Ph.D. Thesis, Lund University of Technology, Sweden.

Fossa M., 2016. Correct design of vertical borehole heat exchanger systems through the improvement of the ASHRAE method. Science and Technology for the Built Environment. 00, 1-10.

Go, G., S.R. Lee, H.B. Kang, S. Yoon and M.J. Kim. 2015. A novel bybrid design algorithm for spiral coil energy piles that considers groundwater advection, Applied Thermal Engineering 78: 196-208.

Hermans, T., F. Nguyen, T. Robert and A. Revil. 2014. Geophysical methods for monitoring temperature changes in shallow low enthalpy geothermal systems, Energies 7: 5083-5118.

Ikard, S.J. and A. Revil. 2014. Self-potential monitoring of a thermal pulse advecting through a preferential flow path, Journal of Hydrology 519 A: 34-49.

Ingersoll, L.R., O.J. Zobel and A.C. Ingersoll. 1954. Heat conduction with engineering, geological, and other applications, McGrawHill, New York.

Lamarche, L. and B. Beauchamp. 2007. A new contribution to the finite line-source model for geothermal boreholes, Energy Build. 39: 188-198. 
Man, Y., H. Yang, N. Diao, J. Liu and Z. Fang. 2010. A new model and analytical solutions for borehole and pile ground heat exchangers, Int. J. Heat and Mass Transfer 53: 2593-2601.

Man, Y., H. Yang, N. Diao, P. Cui, L. Lu and Z. Fang. 2011. Development of spiral heat source model for novel pile ground heat exchangers, HVAC\&R Research 17:6, 1075-1088.

Moch, X., M. Palomares, F. Claudon, B. Souryri and B. Stutz. 2014. Geothermal Helical heat exchangers: comparison and use of twodimensional axisymmetric models, Applied Thermal Engineering 73: 689-696.

Priarone. A. and M. Fossa. 2016. Temperature Response Factors at Different Boundary Conditions for Modelling the Single Borehole Heat Exchanger, Applied Thermal Engineering 103 934-944

Yavuzturk C. and Spitler J.D., 1999. A Short Time Step Response Factor Model for Vertical Ground Loop Heat Exchangers. ASHRAE Transactions, 105, 475-485. 